\title{
An effective drinking device for cats*
}

\author{
R. DAVID STURGEON \\ Tennessee A. and I. State University, Nashville, Tennessee 37203 \\ PATRICK D. BROPHY \\ Division of Humanities and Social Sciences, Rose-Hulman Institute of Technology, Terre Haute, Indiana 47803 \\ and \\ ROBERT A. LEVITT \\ Southern Illinois University, Carbondale, Illinois 62901
}

\begin{abstract}
An effective drinking device has been developed for use with cats. The construction of this device is detailed, and its characteristics are reviewed.
\end{abstract}

Investigation of ingestive behavior requires reliable quantification of that behavior. Small animals, such as rodents, have been studied extensively, and apparatus as well as some techniques have been established, which have aided laboratory investigation of some of these behaviors. At least for rodents whose environment is the laboratory, consummatory responses usually include eating small food pellets or ground up pellets from a food dish and drinking water from a spout. For these animals, then, eating and drinking may be easily quantified.

New apparatus and methodology must be devised for other species when quantifying amounts of food or water consumed. When we decided to investigate thirst in the cat, reliable quantification of water ingestion was one of the first problems encountered. Observation of cats drinking indicated a few milliliters of spillage due to vigorous lapping was to be expected. Quantification of volumes drunk was possible only over relatively long periods of time since drinking bouts would have to be interrupted for measurement using the water dish mounted on the front of the cage. Using this rather crude technique, the water was poured from the dish into a graduated cylinder. Cylinders available for fluid volumes of the $300-$ to $500-\mathrm{ml}$ range are usually calibrated in 5-ml steps. This permits only estimation of points between two calibration lines and is a considerable source of experimental error. A second, and perhaps larger, source of experimental error was the volume of water that adhered to the sides of the water dish due to surface tension.

\section{METHOD}

Several designs for water delivery devices were tested in comparison with the water dish. The most satisfactory turned out to be the tube depicted in Fig. 1. The device is a Kimax tube

*This work was supported by USPHS Research Grant MH-14381, and by the Office of Research and Projects of Southern Illinois University. originally designed for use with rats. Modification of the original tube was done by a glassblower. To facilitate filling and cleaning the tube, a hole was constructed in the top into which fit a No. 3 rubber stopper. The spout of the original tube was replaced by one of larger diameter, and a small drinking cup was fashioned into the end of this spout. The cup had a diameter of approximately $32 \mathrm{~mm}$ at the top due to flaring, but about $25 \mathrm{~mm}$ at the water level. The tube was fastened to the front of the cage with clamps and a stainless steel water-bottle spring may be added for additional security. The tube is calibrated in 1-ml intervals allowing for much greater accuracy while measuring water consumption, and readings may be obtained at any time interval without disturbing the animal's drinking bout.

\section{RESULTS AND DISCUSSION}

The volume of water that evaporated from the tube

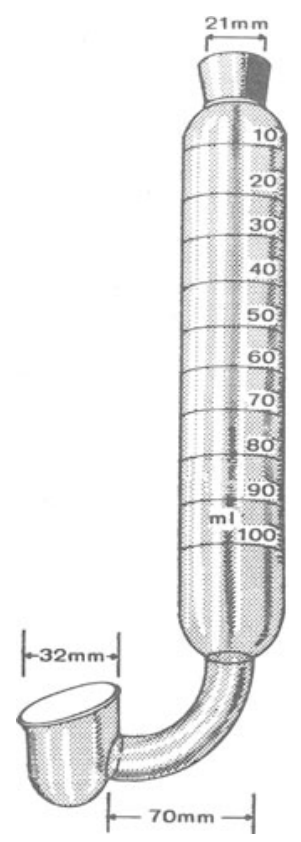

Fig. 1. A drinking device for cats. 
was much less than that from the large water dish. Measurement of evaporation for the two devices was done in the same area of the laboratory. Air flow was approximately equal for all devices and the temperature ranged between $21^{\circ} \mathrm{C}$ and $23^{\circ} \mathrm{C}$. Evaporation from 15 tubes for a 10-day period averaged $.98 \mathrm{ml}$ per day per tube. During the same 10-day period, evaporation from five water dishes was checked. Due to the inherent property of surface tension, evaporation from the dishes was not checked each day, but at the end of the 10-day period. The volume of water lost during this period averaged $17.3 \mathrm{ml}$ per day per dish. The cross-sectional area of the water in the drinking cup on the end of the tube was approximately $4.9 \mathrm{sq} \mathrm{cm}$ while that of the water dish was approximately $78.5 \mathrm{sq} \mathrm{cm}$. This greatly reduces another source of experimental error when using the tube described here.

The drinking tube has, then, several advantages over the water dish. Evaporation is greatly reduced, quantification is more accurate since the tube is graduated in 1-ml intervals, and readings may be obtained at any point in time. The basic tube is improved with the addition of the hole in the top for cleaning and filling.

The drinking behavior of the cat appears to be quite normal. The animal usually learns to drink from the tube within $24 \mathrm{~h}$. Importantly, perhaps in terms of ethology, the response is still one of lapping from a pool of water and not one of licking at a small hole in a spout.

Relatively little research concerning substrates of consummatory behaviors in cats has been conducted. Studies are now being conducted in this laboratory of drinking in this species elicited subsequent to intracranial microinjection of the hormone angiotensin employing the drinking device described here and depicted in Fig. 1 (Brophy \& Levitt, ${ }^{1}$; Sturgeon, Brophy, \& Levitt, 1973; Sturgeon \& Levitt ${ }^{2}$ ).

\section{REFERENCE}

Sturgeon, R. D., Brophy, P. D., \& Levitt, R. A. Drinking elicited by intracranial microinjection of angiotensin in the cat. Pharmacology, Biochemistry \& Behavior, 1973, 1, 353-355.

\section{NOTES}

1. Brophy, P. D. \& Levitt, R. A. Angiotensin-induced drinking in the cat: A biochemical analysis. Submitted for publication.

2. Sturgeon, R. D., \& Levitt, R. A. Angiotensin-induced drinking in the cat: A neuroanatomical analysis. Submitted for publication.

(Received for publication March 2, 1974.) 